\title{
ESTUDO EXPERIMENTAL DO TRATAMENTO DE INCLUSÕES COM CÁLCIO EM ESCALA LABORATORIAL
}

\author{
Wagner Viana Bielefeldt ${ }^{\prime}$ \\ Leomar Marcon ${ }^{2}$ \\ Antônio Cezar Faria Vilela ${ }^{3}$
}

\section{Resumo}

O cálcio (ou ligas a base de cálcio) é muito importante na metalurgia de muitos tipos de aço. Esse elemento é adicionado para promover a desoxidação, dessulfuração, controle da forma de inclusões e para o aumento da lingotabilidade dos aços no lingotamento contínuo. Apesar da importância do uso do cálcio na metalurgia dos aços, o conhecimento acerca do seu comportamento termodinâmico e cinético continua sendo tópico de estudo. Os objetivos deste trabalho são: I) avaliar a evolução da composição química de inclusões modificadas com a adição de cálcio no banho ao longo do tempo de um aço para construção mecânica (SAE 8620); e 2) consolidar uma metodologia para estudo de inclusões em escala laboratorial. Foram executadas corridas em um forno elétrico resistivo com capacidade de até $8 \mathrm{~kg}$ de aço. As matérias-primas utilizadas foram: ferro com alta pureza, ferroligas, alumínio e $\mathrm{CaSi}$. $\mathrm{Na}$ análise química do aço foram considerados os elementos de liga de rotina de produção, bem como o teor de cálcio e oxigênio total. Nas análises de inclusões foram avaliadas: composição química, morfologia, distribuição de fases e tamanho via MEV/EDS. Observou-se a modificação química das inclusões com a adição de cálcio, formando diferentes tipos de cálcio-aluminatos, como previsto. Devido à presença de enxofre verifica-se a formação de sulfetos de cálcio e manganês associados aos cálcio-aluminatos. Palavras-chave: Aço SAE 8620; Caracterização; Cálcio; Inclusões.

\section{EXPERIMENTAL STUDY OF INCLUSION CALCIUM TREATMENT IN LABORATORIAL SCALE}

\begin{abstract}
Calcium (or calcium alloys) is very important in the metallurgy of many kinds of steel. This element is added to promote deoxidation, desulfurization, control of shape of the inclusions and to improve steel castability in continuous casting. Despite of the importance of calcium utilization in metallurgy, the knowledge concerning kinetic and thermodynamic behavior of calcium is still being topic of study. The aims of this work are: I) to evaluate the chemical composition evolution of inclusions modified with calcium addition in the engineering steel bath (SAE 8620) along the time; and 2) to consolidate a methodology for inclusion study in laboratorial scale. Heats were carried out in an electric resistive furnace with capacity of $8 \mathrm{~kg}$ of steel. The raw materials used were: high purity iron, iron-alloys, aluminium and $\mathrm{CaSi}$. In the chemical analysis the routine alloys elements of steelmaking, as well as the oxygen and total calcium content were considered. In the inclusion analysis, the following were considered: chemical composition, morphology, phase distribution and size via SEM/EDS. The chemical modification of inclusions with calcium was observed, forming different types of calcium aluminates, as anticipated. Due to the presence of sulphur, the calcium and manganese sulphites formation associated with calcium aluminates is observed.
\end{abstract}

Key words: SAE 8620; Characterization; Calcium; Inclusions.

\section{INTRODUÇÃO}

Nos aços acalmados ao alumínio, o cálcio é usado com o objetivo primário de modificar as inclusões de alumina para cálcioaluminatos de menor ponto de fusão e maior deformabilidade. As adições de ligas à base de cálcio são usadas para controlar a composição, a distribuição e a morfologia das inclusões remanescentes no aço. $O$ maior benefício desta prática está em minimizar a ocorrência de clogging na etapa de lingotamento contínuo. A modificação de inclusões de alumina sólida em cálcio-aluminatos líquidos (ou parcialmente líquidos) reduz significativamente esse problema. Outros benefícios são a melhoria nas propriedades mecânicas dos aços, em geral, como tenacidade e ductilidade.

'Eng. Metalúrgico, Mestre e Doutorando do Programa de Pós-Graduação em Engenharia Metalúrgica, de Minas e Materiais - PPGEM, Universidade Federal do Rio Grande do Sul - UFRGS, Av. Bento Gonçalves, 9500, CEP 9150I-970, CP I502I, Bairro Agronomia, Porto Alegre, RS, Brasil, e-mail:wagner@ct.ufrgs.br.

2Eng. Metalúrgico, Mestre, Av. Bento Gonçalves, 9500, CEP 91501-970, CP I502I, Bairro Agronomia, Porto Alegre, RS, Brasil, e-mail: vilela@ufrgs.br. 3Prof. Dr.-Ing. DEMET e PPGEM/UFRGS, Av. Bento Gonçalves, 9500, CEP 91501-970, CP I502 I, Bairro Agronomia, Porto Alegre, RS, Brasil, e-mail: vilela@ufrgs.br. 
É de conhecimento geral que o cálcio é difícil de ser usado por ser altamente reativo e ter baixa solubilidade no aço. Desta forma, diferentes modelos termodinâmicos ${ }^{(1)}$ e $\operatorname{cinéticos}^{(2-5)}$ são propostos na literatura para explicar a modificação das inclusões por esse elemento. Levando em conta essas considerações, o presente trabalho tem como objetivos: I) avaliar, em escala laboratorial, a evolução da composição química de inclusões modificadas com a adição de cálcio, ao longo do tempo de ensaio, no banho de um aço para construção mecânica; e 2) consolidar uma metodologia para estudo de inclusões em escala laboratorial.

\section{CONDIÇÕES EXPERIMENTAIS}

\section{I Aparato Experimental e Testes de Produção de Aço}

Uma foto e um esquema do aparato experimental podem ser vistos na Figura I. O forno consiste basicamente de um tubo de grafita, vertical, diâmetro de $145 \mathrm{~mm}$, incluso em uma resistência de grafita. $\mathrm{O}$ aparato está incluso em uma câmara composta por isolante térmico. O tubo e a câmara recebem injeção de argônio de alta pureza (dados típicos: tubo $100 \mathrm{~L} / \mathrm{min}$, câmara $200 \mathrm{~L} / \mathrm{min}$ ).

$O$ forno tem como características relevantes: temperatura máxima de operação: $2.100^{\circ} \mathrm{C}$, temperatura contínua de operação: $1.950^{\circ} \mathrm{C}$; vácuo máximo: $10^{-5} \mathrm{mbar}$; potência aparente: $18 \mathrm{kVA}$; potência ativa: $16 \mathrm{~kW}$; tensão de operação (no primário) $3 \times 380 \mathrm{~V} / 50 \mathrm{~Hz}-60 \mathrm{~Hz}$. O presente trabalho foi conduzido usando tubo de grafita e cadinho de alumina com $99 \%$ de pureza com 2 litros de volume.
O aço escolhido para estudo foi o SAE 8620, cuja composição química dos testes realizados está apresentada na Tabela I. É um aço comumente empregado no estado cementado em peças para a indústria automotiva, aviões, máquinas operatrizes, entre outros. A evolução da composição química ao longo do processo de elaboração na aciaria foi estudada em outros trabalhos dos presentes autores. ${ }^{(6-8)}$

Foram realizados três ensaios, nos quais foram analisadas seis amostras por ensaio, mais - aço restante no cadinho. Os testes foram chamados de Teste I, Teste 2 e Teste 3 (Tabela I). O procedimento experimental seguiu as seguintes etapas: I) Preparação da carga ( $\mathrm{kg}$ para o teste I; I kg para o teste 2; e 2 kg para o teste 3); 2) programação de aquecimento $\left(T=1.627^{\circ} \mathrm{C}\right)$ e resfriamento, via controle CLP do forno; 3) retirada de amostras do aço líquido; 4) adição de $\mathrm{Al}$ e CaSi; 5) retirada de amostras do aço líquido; 6) resfriamento do forno; 7) preparação das amostras; 8) análises. A Figura 2 ilustra a metodologia do ensaio indicando os pontos de amostragem. As composições relatadas na Tabela I correspondem ao ponto de amostragem amostra final.

A carga foi preparada a partir da mistura de ferro de alta pureza com as ligas comumente usadas na indústria para a produção do aço SAE 8620. Essas ligas são: grafita; ferro-silício (FeSi75); Mn eletrolítico; Ni eletrolítico; enxofre;
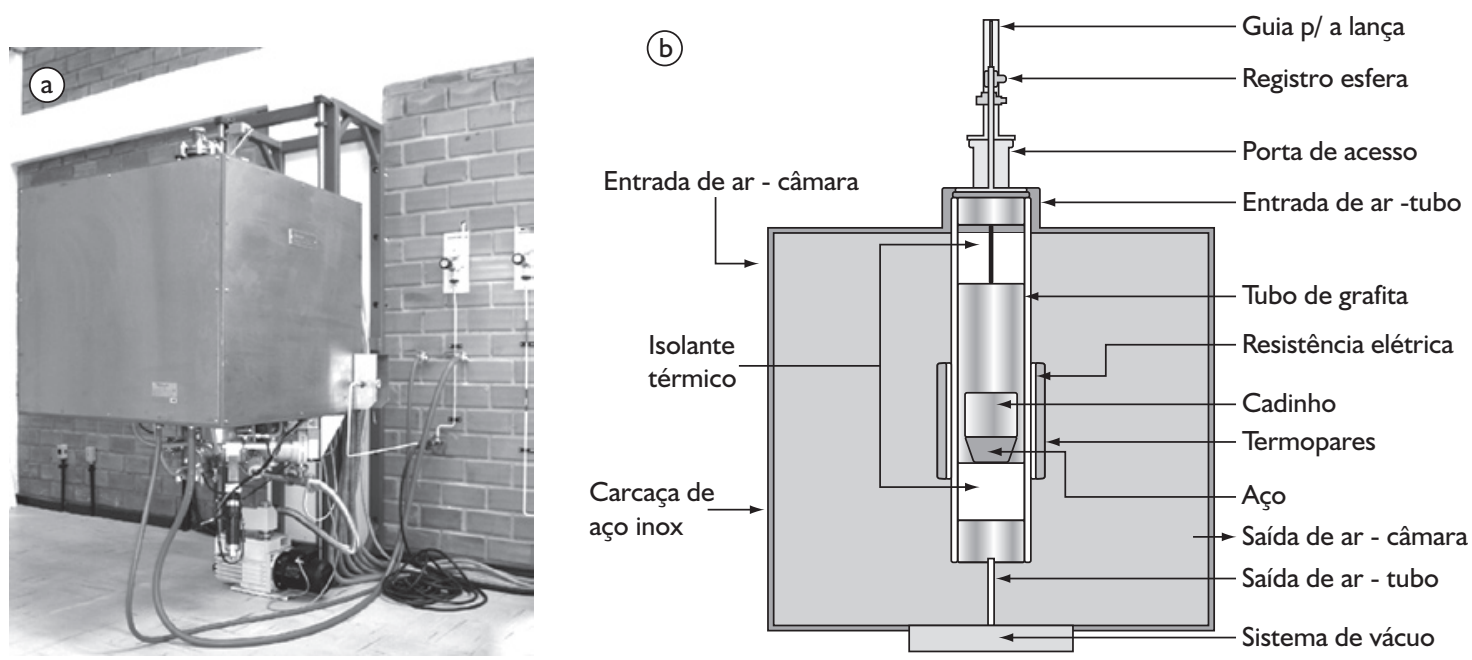

Figura I. a) Foto do forno elétrico resistivo. b) esquema do forno.

Tabela I. Composição química para o aço SAE 8620 em \% em massa.

\begin{tabular}{lcccccccccccc}
\hline & $\mathbf{C}$ & $\mathbf{S i}$ & $\mathbf{M n}$ & $\mathbf{P}$ & $\mathbf{S}$ & $\mathbf{C r}$ & $\mathbf{N i}$ & $\mathbf{M o}$ & $\mathbf{A l}_{\text {total }}$ & $\mathbf{C a}^{*}$ & $\mathbf{N}_{\mathbf{2}} *$ & $\mathbf{O}_{\text {total }}{ }^{*}$ \\
\hline Teste I & 0,18 & 0,24 & 0,88 & 0,007 & 0,035 & 0,63 & $0,5 \mathrm{I}$ & 0,007 & 0,019 & 5 & 22 & 20 \\
Teste 2 & 0,24 & 0,25 & 0,89 & 0,008 & 0,019 & 0,52 & $0,5 \mathrm{I}$ & 0,007 & 0,021 & 7 & 18 & 24 \\
Teste 3 & 0,26 & 0,22 & 0,86 & 0,007 & 0,017 & 0,59 & $0,5 \mathrm{I}$ & 0,007 & 0,027 & 8 & 22 & 20 \\
\hline
\end{tabular}

* $\mathrm{Ca}, \mathrm{N}_{2}$ e $\mathrm{O}_{\text {total }}$ em ppm. $\mathrm{Mg} \approx \mathrm{Ippm}$. 


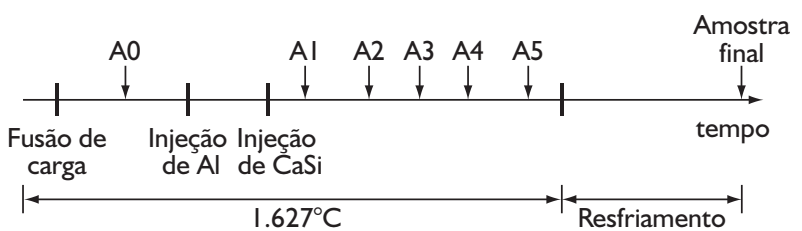

Figura 2. Esquema dos ensaios indicando os pontos de amostragem, A0 até a amostra final.

ferro-cromo com baixo carbono. Oxigênio foi incorporado à carga na forma de $\mathrm{Fe}_{2} \mathrm{O}_{3}$. Foram coletadas amostras - na forma de pinos - pesando aproximadamente $8 \mathrm{~g}$ cada - antes e após a adição de Al e CaSi. Utilizaram-se amostradores de sucção de quartzo, encontrados no mercado.

\subsection{Análises Realizadas}

Para avaliar a composição química final do aço, das inclusões e oxigênio total, foram realizadas análises na seção transversal das amostras de aço remanescente no cadinho. Nos pinos, foram realizadas somente análises de inclusões. Para a composição química final do aço foi usado um espectrômetro de emissão óptica. Para o teor de oxigênio total, usou-se um determinador simultâneo de oxigênio e nitrogênio LECO. A análise da composição química das inclusões, bem como os mapas de raios $\mathrm{X}$, foram realizados em um microscópio eletrônico de varredura (MEV) com EDS acoplado e elétrons retroespalhados.

\section{RESULTADOS E DISCUSSÃO}

Neste capítulo são apresentados e discutidos os resultados obtidos das análises realizadas. Nem todos os testes estão representados em cada tópico. Isso porque, estatisticamente (via comparação dos grupos - One-way Analysis of Variance), verificou-se que não houve diferença significativa entre os testes.

\section{I Análise de Inclusões Via MEV/EDS}

$\mathrm{Na}$ amostra TIA0, antes das adições de $\mathrm{Al}$ e $\mathrm{CaSi}$, foram encontrados dois diferentes tipos de inclusões. Elas foram classificadas em dois grupos: um contendo $\mathrm{CaO}$, chamado de $\mathrm{TIAOcCa} e$ outro não contendo $\mathrm{CaO}$, chamado de TIAOsCa, apresentadas na Figura 3. $\mathrm{Na}$ amostra TIAO, as inclusões enquadram-se no sistema $\mathrm{CaO}$ (ou $\mathrm{MnO}$ ) $-\mathrm{Al}_{2} \mathrm{O}_{3}-\mathrm{SiO}_{2}$. Importante salientar que o grupo de inclusões sem cálcio representa aproximadamente $80 \%$ das inclusões analisadas na prova $\mathrm{A} 0$.

A desoxidação dos aços com Si/Mn produz inclusões do tipo $\mathrm{MnO}-\mathrm{SiO}_{2}$; porém, na presença de outros desoxidantes fortes como $\mathrm{Al}$ e $\mathrm{Ca}$, mesmo que em pequenos teores, aparecem agregados a esse tipo de inclusão os óxidos desses respectivos desoxidantes. ${ }^{(9)}$ A principal fonte desses elementos residuais está na liga $\mathrm{FeSi75}$, que possui como impurezas aproximadamente I, $1 \%$ de $\mathrm{Ca}$ e I, $4 \%$ de Al.

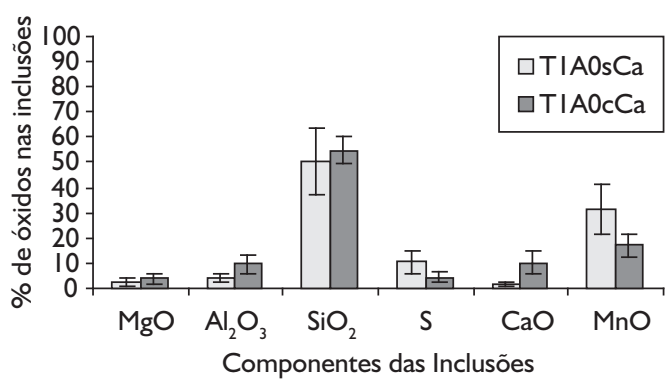

Figura 3. Média e desvio-padrão da \% em massa dos componentes das inclusões analisadas na $\mathrm{TI}-\mathrm{A} 0$, sem(s) e $\operatorname{com}(c)$ a presença de $\mathrm{CaO}(\mathrm{Ca})$.

O histograma da Figura 4 apresenta a média e o desvio-padrão dos componentes das inclusões analisadas para as amostras do Teste I, após o tratamento com Al e CaSi. São as amostras $A I$ até $A 4$, sendo que $A l$ corresponde a cerca de 2 minutos após a adição de CaSi. Na Figura 5 estão agrupados os resultados da prova final (aço solidificado no forno) para os três ensaios. Em ambas as Figuras 4 e 5 as inclusões enquadram-se no sistema ternário $\mathrm{CaO}-\mathrm{Al}_{2} \mathrm{O}_{3}-\mathrm{MgO}$, com traços de $\mathrm{SiO}_{2}$ e $\mathrm{MgO}$.

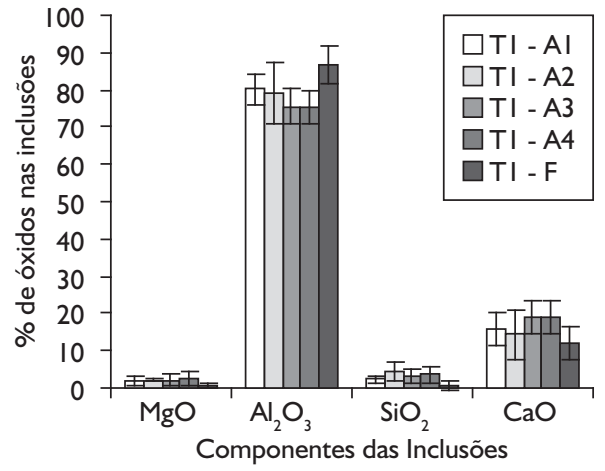

Figura 4. Média e desvio-padrão da \% em massa dos componentes das inclusões analisadas no teste I, após o tratamento com Al e CaSi.

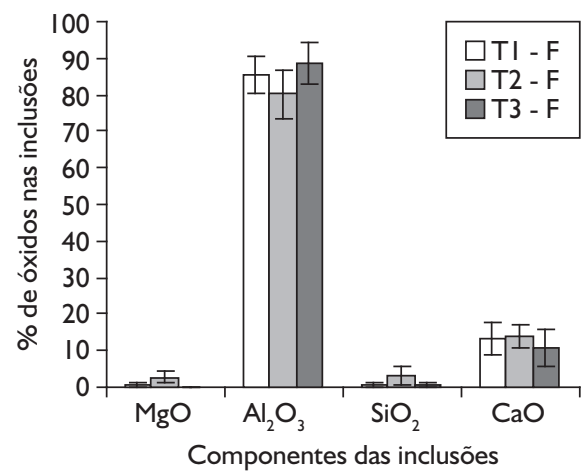

Figura 5. Média e desvio-padrão da \% em massa dos componentes das inclusões nas amostras finais (F). 
Nota-se que as inclusões evoluem do sistema $\mathrm{MnO}-\mathrm{SiO}_{2}$ (Figura 3) para cálcio-aluminatos ricos em alumina (entre $\mathrm{CaO} .6 \mathrm{Al}_{2} \mathrm{O}_{3}$ e $\mathrm{CaO} .2 \mathrm{Al}_{2} \mathrm{O}_{3}$ ) (Figuras 4 e 5 ). $\mathrm{O}$ baixo rendimento do cálcio pode ser atribuído ao fato dos experimentos terem sido conduzidos sem a presença de escória. Das et al. ${ }^{(10)}$ afirmam que uma grande quantidade de CaSi deve ser usada para a modificação de inclusões quando for usado sem escória. Segundo os autores, a influência positiva da escória está relacionada ao fato da evaporação do cálcio ser minimizada pela escória. A liga de cálcio é dispersa na escória a qual, provavelmente, dá um efeito protetivo. A concentração de cálcio no banho é fortemente influenciada pelas reações de vaporização durante e após o tratamento com cálcio.( ${ }^{(1)} \mathrm{O} \mathrm{Mg}$, praticamente residual, é proveniente como impureza da liga de Al.

A Figura 6 relaciona a composição média das inclusões (em termos de $\% \mathrm{CaO}$ e $\% \mathrm{Al}_{2} \mathrm{O}_{3}$ ) no banho de aço antes e depois das adições de $\mathrm{Al}$ e $\mathrm{CaSi}$ com o tempo. A faixa entre -4 e -2 minutos corresponde à adição de $\mathrm{Al}$. $\mathrm{O}$ tempo zero corresponde à adição de $\mathrm{CaSi}$. Os pontos antes do tempo zero correspondem à amostra $\mathrm{A} 0$. Os pontos após o tempo zero correspondem às amostras Al até A4.

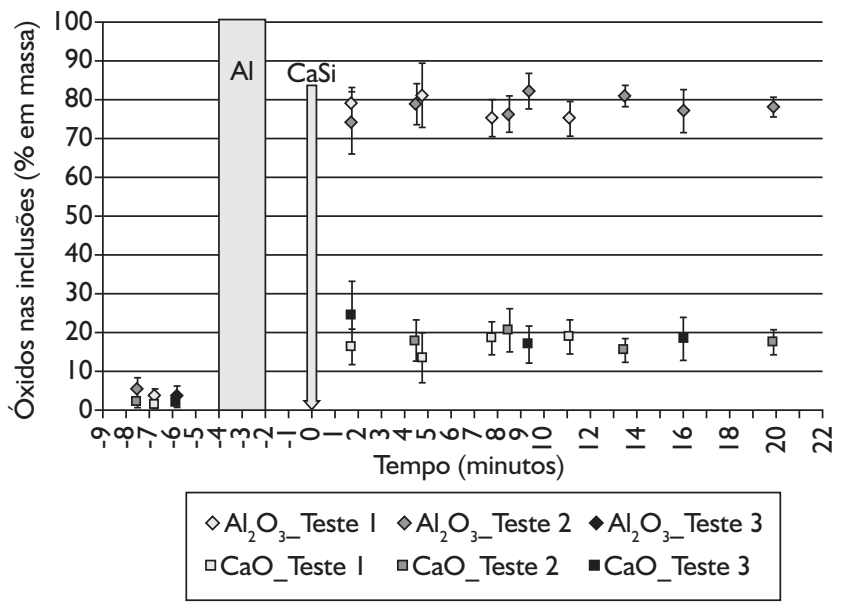

Figura 6. Teores de $\mathrm{CaO}$ e $\mathrm{Al}_{2} \mathrm{O}_{3}$ nas inclusões ao longo do tempo de ensaio.

Pela análise da Figura 6, verifica-se que os percentuais de $\mathrm{CaO}$ e $\mathrm{Al}_{2} \mathrm{O}_{3}$ nas inclusões permanecem constantes, desde 2 minutos após o tratamento com cálcio, até o final do experimento (20 min). Lu et $\mathrm{al}^{(2)}$, em um estudo sobre a cinética de modificação de inclusões com cálcio, observam que um minuto após a adição de cálcio já não é mais possível verificar a presença de inclusões de alumina, havendo apenas cálcio-aluminatos presentes no banho.

\subsection{Distribuição de Tamanhos das Inclusões via MEV}

A Figura 7 mostra o tamanho médio das inclusões por amostra analisada via MEV. Dois minutos após a injeção de CaSi, as inclusões de cálcio-aluminatos apresentam um tamanho que se mantém constante com o tempo. Essa distribuição de tamanhos foi observada nos experimentos em laboratório conduzidos por outros autores, ${ }^{(11,12)}$ e também em amostras coletadas na planta ${ }^{(7)}$. $O$ aumento do diâmetro médio nas amostras $F$ se deve à precipitação dos compostos de $(\mathrm{Ca}, \mathrm{Mn}) \mathrm{S}$ na superfície das inclusões durante a solidificação lenta, como mostram os mapas de raios X (Item 3.3).

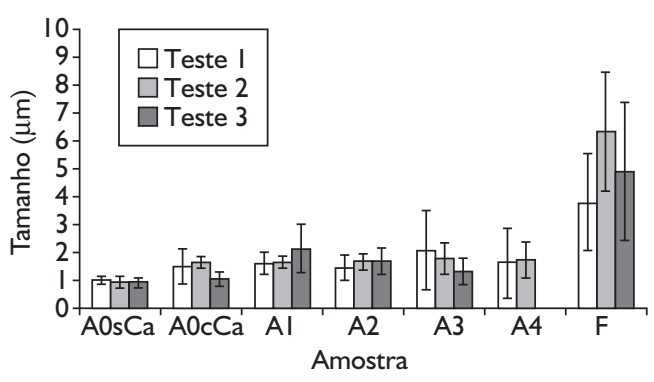

Figura 7. Média e desvio-padrão de tamanhos das inclusões analisadas nos ensaios.

\subsection{Análise de Inclusões via Mapas de Raios X}

As Figuras 8 e 9 apresentam mapas de raios $X$ de inclusões características das amostras retiradas do aço líquido e do aço resfriado no cadinho, respectivamente. No primeiro caso, observa-se que são homogêneas e esféricas, portanto líquidas a $1.627^{\circ} \mathrm{C}$. No segundo caso, no qual a solidificação se procedeu de maneira lenta, aparecem compostos formados por ( $\mathrm{Ca}, \mathrm{Mn}) \mathrm{S}$ na superfície dos cálcio-aluminatos.

\section{CONCLUSÕES}

Neste trabalho é avaliada, em escala laboratorial, a evolução de inclusões com o tratamento com cálcio para o aço SAE 8620.

É observado claramente o efeito das adições de alumínio e cálcio na composição das inclusões. Inclusões esféricas de $\mathrm{MnO}-\mathrm{SiO}_{2}$ são modificadas para inclusões no sistema $\mathrm{CaO}-\mathrm{Al}_{2} \mathrm{O}_{3}$, esféricas e quimicamente homogêneas. Não se observa mudança na composição das inclusões ao longo dos ensaios.

As inclusões apresentam um tamanho médio de I $\mu \mathrm{m}$ a $2 \mu \mathrm{m}$, constante com o tempo de ensaio. $O$ tamanho médio aumentou na amostra final, claramente devido à precipitação de sulfetos ao redor das inclusões de óxido.

Também é observada a formação de (Ca,Mn)S ao redor das inclusões, na solidificação da amostra no cadinho, via mapas de raios $X$.

\section{Agradecimentos}

Os autores agradecem à Gerdau Aços Especiais Piratini pela importante parceria estabelecida ao longo dos anos para o desenvolvimento de conhecimento tecnológico em siderurgia. Wagner V. Bielefeldt agradece ao CNPq pelo financiamento de sua bolsa de doutorado. 

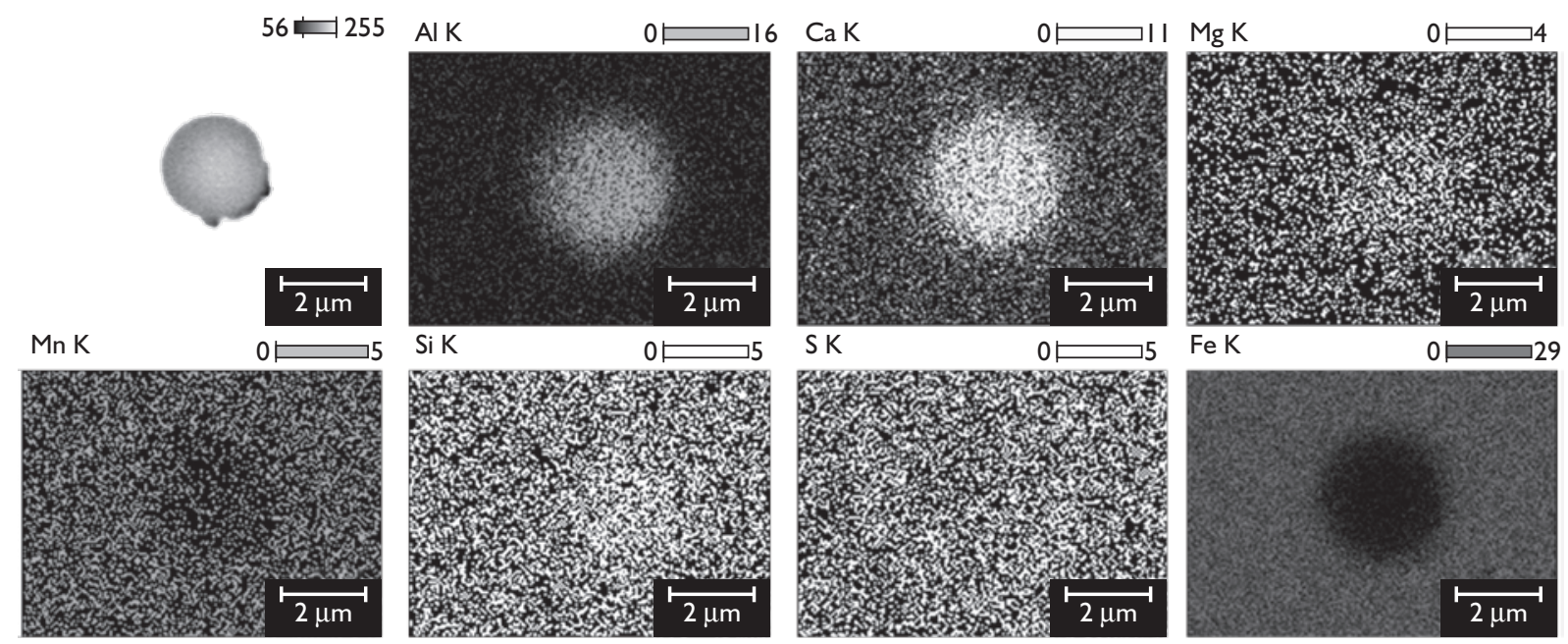

$\mathrm{Fe} \mathrm{K}$

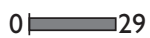

Figura 8. Mapas de raios $X$ característicos de uma inclusão da amostra T2-AI.
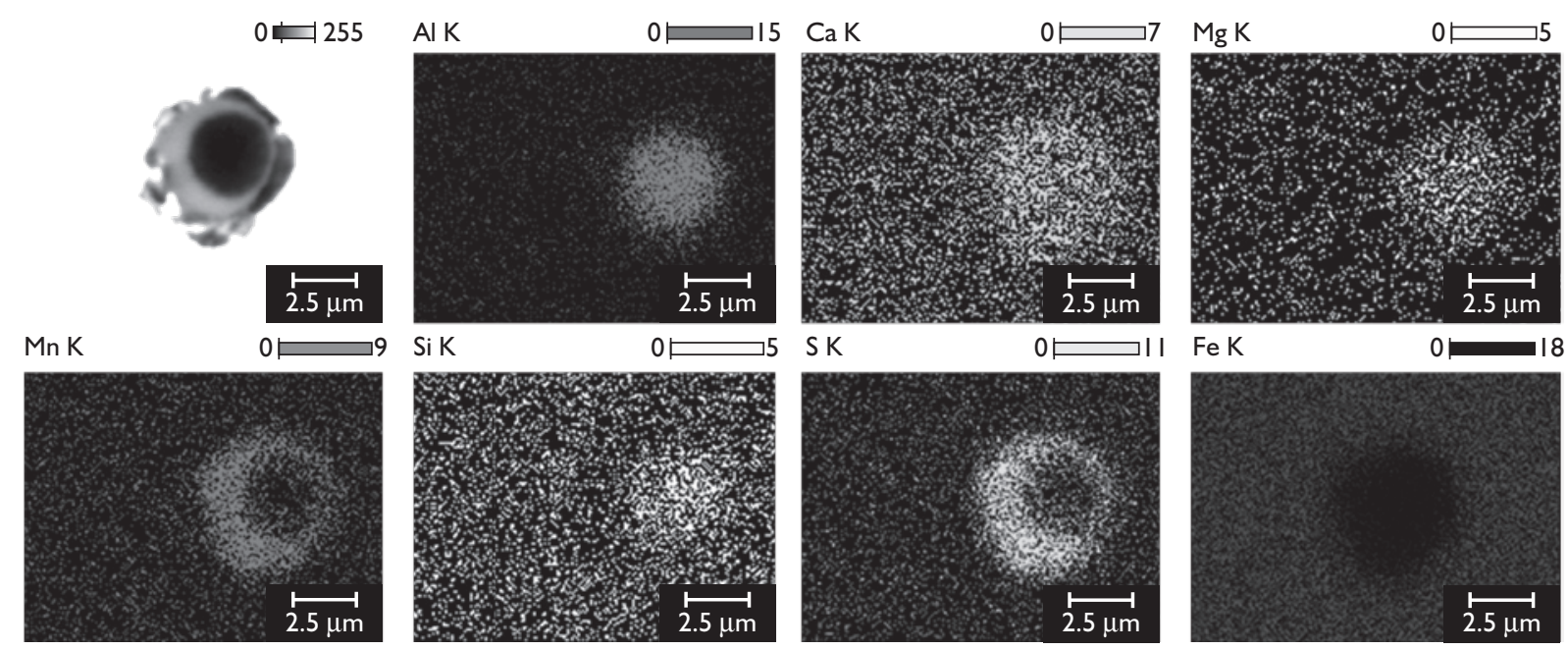

Figura 9. Mapas de raios $\mathrm{X}$ característicos de uma inclusão da amostra T2-F.

\section{REFERÊNCIAS}

I SILVA, A.C. Calcium and magnesium thermodynamics in steel and its impacts on secondary steelmaking: a computational thermodynamic approach. La Revue de Métallurgie CIT, v. I05, n. 4, p. I8I-93, avril 2008.

2 LU, D.-Z.; IRONS, G. A.; LU, W.-K. Kinetics and mechanisms of calcium dissolution and modification of oxide and sulphide inclusions in steel. Ironmaking and Steelmaking, v. 21, n. 5, p. 362-7I, 1994.

3 ITO, Y.; SUDA, M.; KATO, Y.; NAKATO, H.; SOMIRACHI, K. Kinetics of shape control of alumina inclusions with calcium treatment in line pipe steel for sour service. ISIJ International, v. 36, supl., p. SI48-50, 1996.

4 PARK, J. H.; KIM, D.S.; LEE, S-B.; LEE, Y.Y. Inclusion control of $\mathrm{Fe}-16 \% \mathrm{Cr}$ stainless steel melts by aluminium deoxidation and calcium treatment. AIST Transaction, v. 4, n. I, p. I38-45, Jan. 2007.

5 HOLAPPA, L.; LIND, M.; LIU, L.; HAN, Z. J. Mechanism and kinetics of transformation of alumina inclusions by calcium treatment. Acta Metallurgica Sinica, v. 19, n. I, p. I-8, Feb. 2006.

6 BIELEFELDT, W. V.; VILELA, A. C. F.; MORAES, C. A. M.; KLUJSZO, L. A. C.; FERNANDES, P. C. Evolução das inclusões no processamento do aço SAE 8620 via aciaria elétrica. In: CONGRESSO ANUAL DA ABM, 59., 2004, São Paulo. Anais... São Paulo: ABM, p. 736-45, 2004. 
7 BIELEFELDT, W. V. Estudo do tratamento de inclusões com cálcio na fabricação do aço SAE 8620. 2005. 134p. Dissertação (Mestrado em Engenharia Metalúrgica) - Universidade Federal do Rio Grande do Sul, Porto Alegre, 2005.

8 BIELEFELDT, W. V.; VILELA, A. C.F.; MORAES, C. A. M.; FERNANDES P. C. Computational thermodynamics application on the calcium inclusion treatment of the SAE 8620 steel. Steel Research International, v. 78, n. 12, p. 857-62, Dec. 2007.

9 MARCON L.; BIELEFELDT, W. V.; MORAES, C. A. M.; VILELA, A. C. F. Uso de diferentes técnicas para caracterização de inclusões não-metálicas em aços. In: IAS STEELMAKING CONFERENCE, I5. Proceedings... San Nicolas, Argentina. p. 707-14, Nov. 2005.

10 DAS, N. K.; SEN, N.; GHOSH, M.; SAU, R. Effect of simultaneous addition of CaO-Al2O3 flux and CaSi on the modification of inclusions in aluminium-killed steel. Scandinavian Journal of Metallurgy, v. 34, n. 5, p. 276-82, 2005.

I I HIGUCHI, Y.; NUMATA, M.; FUKAGAWA, S.; SHINME, K. Inclusion modification by calcium treatment. ISIJ International, v. 36, supl., p. SI5I-48, 1996.

12 ANDERSSON, M.; BERLIN, D.; JÖNSSON, P.; LÖWNERTZ, M. The influence of different calcium-based additions on desulphurisation and inclusion characteristics. Scandinavian Journal of Metallurgy, v. 30, n. 2, p. I27-35, 200 I.

Recebido em: 25/03/08

Aceito em: 0I/09/08

Proveniente de: SEMINÁRIO DE ACIARIA - INTERNACIONAL, 38.., 2007, Belo Horizonte, MG. São Paulo: ABM, 2007. 